add up to a human on a chip. Each one of them lacks critical components to be a full tissue, but it still can be tremendously useful," he said. If the goal is to improve evaluation of the effects of chemical exposures, Andersen suggested that it may make sense to consider more carefully which tissue systems should be included in the first test systems. Rather than rushing to combine all of the organ systems to produce a "truncated human on a chip," Andersen pointed out that the collaborators may do well to show that a more limited platform can faithfully represent expected tissue exposures in an intact organism.

Suzanne Fitzpatrick, Senior Science Advisor of the FDA's Office of the Commissioner's Office of the Chief Scientist closed the meeting. "In putting together this program with the FDA and CAAT, we wanted to create a community of people who were all working toward a common purpose," she said. She credited the National Research Council's Toxicity Testing in the 21 ${ }^{\text {st }}$ Century report for helping to catalyze the creation of such a community. "To move innovation, we really need a whole community of people... to look at these new toxicology models," she concluded.

A more detailed summary of the workshop is available at: http://www.altex-edition.org

\section{References}

Arrowsmith, J. (2011). Trial watch: Phase II failures: 2008-2010. Nat Rev Drug Discov 10, 328-329. http://dx.doi.org/10.1038/ $\operatorname{nrd} 3439$
Huh, D., Matthews, B. D., Mammoto, A. et al. (2010). Reconstituting organ-level lung functions on a chip Science 328, 1662-1668. http://dx.doi.org/10.1126/science.1188302

Huh, D., Leslie, D. C., Matthews, B. D. et al. (2012). A human disease model of drug toxicity - induced pulmonary edema in a lung-on-a-chip microdevice. Sci Trans Med 4, 159ra147. http://dx.doi.org/10.1126/scitranslmed.3004249

Kim, H. J., Huh, D., Hamilton, G. and Ingber, D. E. (2012). Human gut-on-a-chip inhabited by microbial flora that experiences intestinal peristalsis-like motions and flow. Lab Chip 12, 2165-2174. http://dx.doi.org/10.1039/C2LC40074J

Kim, H. J. and Ingber, D. E. (2013). Gut-on-a-Chip microenvironment induces human intestinal cells to undergo villus differentiation. Integr Biol (Camb) 5, 1130-1140. http://dx.doi. org/10.1039/c3ib40126j

Kola, I. and Landis, J. (2004). Can the pharmaceutical industry reduce attrition rates? Nat Rev Drug Discov 3, 711-716. http://dx.doi.org/10.1038/nrd1470

\section{Correspondence to}

Kellyn Betts

8023 Glenside Drive

Takoma Park, MD 20912

USA

Phone: +1 202.321.6678

e-mail: k_betts@nasw.org

\title{
3Rs and New Frontiers in Laboratory Techniques
}

\author{
Francesca Caloni ${ }^{1}$, Gianni Dal Negro ${ }^{2}$, Patrizia Costa ${ }^{3}$, Silvia Dotti ${ }^{4}$, \\ Giacomo Matteucci $^{5}$, Augusto Vitale ${ }^{6}$ and Isabella De Angelis ${ }^{7}$ \\ ${ }^{1}$ Università degli Studi di Milano, Department of Health, Animal Science and Food Safety (VESPA), Milan, Italy; \\ ${ }^{2}$ GlaxoSmithKline R\&D Limited, United Kingdom; ${ }^{3}$ Università degli Studi di Milano, Laboratory Animal Science and Medicine \\ Specialization School, Milan, Italy; ${ }^{4}$ Istituto Zooprofilattico Sperimentale della Lombardia e dell'Emilia Romagna "Bruno \\ Ubertini”" (IZSLER), Brescia, Italy; ${ }^{5}$ Università degli Studi di Siena, Siena, Italy; ${ }^{6}$ Istituto Superiore di Sanità, ISS, Department of \\ Cell Biology and Neuroscience, Section of Behavioral Neuroscience, Rome, Italy; ${ }^{7}$ Istituto Superiore di Sanità, ISS, Department \\ of Environment and Primary Prevention, Rome, Italy
}

http://dx.doi.org/10.14573/altex.1406182

On May 8, 2014, a meeting on advanced laboratory techniques was held by the Italian Reference Center for Alternative Methods, Welfare and Care of Laboratory Animals. The meeting, hosted by the Istituto Zooprofilattico Sperimentale della Lombardia e Emilia Romagna, was opened by Dr Stefano Cinotti (ISZLER) and chaired by Francesca Caloni, University of Milan and Gianni Dal Negro, GlaxoSmithKline.
The first speaker, Dr Marlies Halder (JRC, Ispra, Italy), provided a comprehensive overview on methods for quality control of vaccines. Halder showed how such methods for new vaccines are almost exclusively based on in vitro tests, while for old vaccines, which have until now been tested using animals, the "consistency approach" is increasingly being embraced, reducing animal use. Halder expressed the hope that Directive 
2010/63/EU will speed up the validation process of alternative methods in the context of vaccines. Julie Holder (GSK, UK) gave a presentation on induced pluripotent stem (iPS) cells in regenerative medicine with a particular focus on cardiomyocytes and provided a state of the art overview of methods currently available for 3D iPS cultures in heart research. Dr George Loizou, (Computational Toxicology Team, Mathematical Sciences Unit, Health \& Safety Laboratory Buxton, UK), shared present and future perspectives in computational modelling applied to toxicology. Loizou emphasized the critical role that human data will play on developing animal free approaches in toxicology by applying the P4 (Predictive, Preventive, Personalized, Participatory) medicine approach. Robin Williams (Centre for Biomedical Science, Royal Holloway, University of London, UK) presented the social amoeba Dictyostelium as a screening model in early drug development. In particular, Williams presented some data on the possible application of this eukaryotic cell model to predict bitter taste of oral formulations and replace the rat pica model. Paolo Bazzicalupo (Institute of Biosciences and Bioresources, Italian National Research Council, Naples, Italy) presented the nematode Caenorhabditis elegans as a model organism for pharmaco-toxicological studies and screening purposes (Kaletta and Hengartner, 2006) and for various human pathologies, in particular neurodegenerative diseases. $C$. elegans is suitable to address fundamental biological questions, Bazzicalupo said, for the following reasons: i) ease of experimental manipulations, ii) anatomy and development described in great detail (it is transparent) and iii) powerful molecular genetics approaches $(>70 \%$ of C. elegans genes have homologs in other organisms and $>55 \%$ of $\mathrm{C}$. elegans genes have a significant human match, including many genes involved in human diseases). In 2006 the US National Toxicology Program started a project to develop medium- throughput toxicity screens using C.elegans.

The afternoon session, chaired by Isabella De Angelis, ISS and Giovanna Lazzari, Avantea, was opened by Dr Thomas Keller (GSK, UK), who spoke on human relevant high content screening platforms based on organ-functionalities-on-a-chip. Tissue chips are engineered microsystems that represent units of human organs, such as lung, liver and heart, modeling both structure and function. This technology aims to improve the reliability of drug development and toxicology screening. In particular, Dr Keller described a human gut-on-a-chip model that mimics the complex structure and physiology of living intestine using human intestinal epithelial cells (Caco-2) maintained under a low rate fluid flow. These conditions accelerate intestinal epithelial cell differentiation, promote formation of 3D villi-like structures and increase intestinal barrier function; the addition of cyclic mechanical strain that mimics normal peristaltic motions, further enhances these responses. Moreover, once differentiated within the gut-on-a-chip device, the intestinal epithelium can support growth of microbial flora that normally lives within the human intestine (as Lactobacillus rhamnosus GG) without compromising its viability (Kim et al., 2012). The human peristaltic gut-on-a-chip may greatly improve the study of the mechanic regulation of intestinal function, as well as hostmicrobe symbiosis and evolution. In his second presentation, Dr Loizou, presented an innovative in silico PBPK model designed to predict tissue concentration and biologically effective dose. PBPK models can support extrapolation across dose-routes, different dose and exposure scenarios and between species; inter-individual variability and sensitive subpopulations also can be represented. PBPK modelling also is successfully used in reverse dosimetry, or dose reconstruction, approaches, where environmental exposure is calculated based on biomarker measurements from blood or urine samples. In vitro data, derived from both cell culture or toxicogenomic assays, may be also utilized. The reverse dosimetry approach has great potential as a tool for risk assessment.

In the round table discussion, participants shared their opinions on relative replacement, distinct from absolute replacement in which animals are not used at all. Despite different interpretations, there seemed to be a consensus that in vivo and in vitro activities are inherently connected and complementary. Participants agreed that relative replacement represents the best compromise at this time, while looking forward to putting an end to our reliance on animals in research activities.

\section{References}

Kim, H. J., Huh, D., Hamilton, G. and Ingber, D. E. (2012). Human gut-on-a-chip inhabited by microbial flora that experiences intestinal peristalsis-like motions and flow. Lab Chip 12, 2165-2174. http://dx.doi.org/10.1039/c2lc40074j

Kaletta, T. and Hengartner, M. O. (2006). Finding function in novel targets: C. elegans as a model organism. Nat Rev Drug Discov 5, 387-399. http://dx .doi.org/10.1038/nrd2031 